\title{
Investigation of Dust Transfer Processes During Loading and Unloading Operations using Software Simulation
}

\author{
Yuriy Dmitrievich Smirnov ${ }^{1}$, Andrey Vladimirovich Ivanov ${ }^{1}$ \\ 1 Saint-Petersburg Mining University, 21 Lines of Vasilyevsky Island, 199106 Saint-Petersburg, Russia \\ * Corresponding author's e-mail: qwerik84@gmail.com
}

\begin{abstract}
In order to limit dust transfer during the operations of material transportation at the factories of the construction industry, factories of the mineral and raw materials complex employ special dust suppression bunkers. These devices are structures located above a railway hopper (either a truck body or a conveyor). Through the input portal of the device, the transported material is transferred. Dusting can also be carried out under the bunkers from the side of the hopper, into which the material is poured. The design of dust suppression bins should be accompanied by a simulation of dust propagation processes. The simulation should be carried out in order to minimize the dust emissions and to select the optimum locations for the dust suppression sprayers.
\end{abstract}

Keywords: bunker, dust suppression, ANSYS, Simulation, ROCKY, hopper

\section{INTRODUCTION}

In order to limit dust transfer during the operations of material transportation at the factories of the construction industry, factories of the mineral and raw materials complex employ special dust suppression bunkers. These devices are structures located above a railway hopper (either a truck body or a conveyor). Through the input portal of the device, the transported material is transferred. At the entrance elastic curtains are installed (one of the effective methods of solution is described in the Patent of the Russian Federation \#2536573), which prevent the dust from escaping into the surrounding air. In addition, the device developed at the Center for Collective Use of the Mining University is equipped with a system of water dust suppression (sprayers, the design of which is described in the patent of the Russian Federation \#2446021). Certainly, some dust can penetrate through the elastic curtains, but their installation reduces the dusting of the fraction which is dangerous to human by $90-95 \%$. Dusting can also be carried out under the bunkers from the side of the hopper, into which the material is poured. The design of dust suppression bunkers should be accompanied by a simulation of dust propagation processes. The simulation should be carried out in order to minimize dust emissions and to select the optimum locations for the dust suppression sprayers.

\section{MATERIAL AND METHODS}

At present, there are no software complexes that allow correct modeling of the dust particles propagation (Volkodaeva, M.V. 2015). The applied calculation methods, such as the Russian OND-86 (versions, both 1987 and 2017) and others, firstly, do not consider the factors influencing the slinging and holding of dust particles in the air; secondly, they do not take into account the effect of the motion of the poured material.

As far as the existing universal software packages are concerned, in recent years, the most popular one is "Ansys" - a universal software system of finite element analysis. This software complex is widely used in the field of computeraided engineering, solving linear and nonlinear, stationary and non-stationary spatial problems of mechanics of deformable solids and structural 
mechanics (including nonstationary geometrically and physically nonlinear problems of contact interaction of structural elements), problems of mechanics liquid and gas, heat transfer and heat transfer, electrodynamics, acoustics, and also the mechanics of coupled fields (Kaplun, et al. 2017).

In the course of the scientific work, the use of "Ansys" complex was suggested, but in combination with the "Rocky" software, which allows modeling the movement of individual particles of the material being poured. Using "Ansys", as well as other programs, does not allow to simulate the dusting processes themselves; therefore, the task is in fact reduced from determining the speed and direction of air movement, which can be interpreted as the dust intensity and the direction of dust propagation (Ivanov, et al.. 2016)

The calculation was carried out in the "Rocky" software on the basis of the discrete elements method (this is the simulation of the interaction of particles and elements of a geometric model) and one of the computational fluid dynamics (CFD) variants - the Lattis-Boltzmann method, or the Boltzmann lattice equation method. The Lattice-Boltzmann method consists in the following: hydro- and aerodynamics are described macroscopically by the Navier-Stokes equation. It shows what the pressure, density and velocity of the liquid will be at each point of space at each instant of time, depending on the initial and boundary conditions and parameters of the medium. On the other hand, for rarefied gases, the Boltzmann equation holds, describes how the particle density distribution of the velocities changes at each point of space with time. Macroscopically, the Boltzmann equation is equivalent to the NavierStokes equation (Fedorova, et al. 2017).

The dust analyzer DustTrak 8533 was used in the studies. The instrument is based on the nephelometric method of analysis, which consists in measuring the intensity of light scattered in a sample of dusty air. A universal meteorometer MES-200A was used in order to determine the speed of air movement.

\section{RESULTS AND DISCUSSIONS}

At present, during design, the overall dimensions and shape of the dust suppression bunkers are selected without evaluating the simulation of dust-transfer processes. The choice of geometric parameters is based on the convenience of installation and the availability of material for the manufacture of the bunker.

In a number of enterprises, dust suppression silos are not used intentionally, since large local dust emissions are possible when using the hopper, which creates negative working conditions for personnel, and without a bunker, the discharge is carried out practically in all directions and a rapid dispersion of dust occurs (Bulbashev, et al. 2009).

Nevertheless, enterprises are forced to make annual payments for the emission of aerosol particles into the atmosphere and for the degradation of soil and vegetation near the production territory.

Figure 1 shows a non-isolated place of pouring at the "Vyborg Career Management" factory. The photographs show that at the time of overloading, dust is released from the receiving portal. Moreover, due to the fact that the site of the crossing is located on the dominant altitude, the dust is instantly picked up by the wind and carried throughout the territory. It should be noted that it involves granite dust with a high content of silicon oxide $\left(\mathrm{SiO}_{2}\right)$, which is the main reason for the occurrence of occupational diseases of workers, i.e. silicosis.

Through the "Ansys" and "Rocky" software a model of the movement of material and movement of air masses, provoked by it, was constructed. The air movement was modeled according to Figure 2 - the space was separated by a grid in cells of which the allowed directions of motion were set and, further, the actions "current-collision" were simulated (Basov, 2016).

Granite with a density of $2600 \mathrm{~kg} / \mathrm{m}^{3}$ was chosen as the starting material. The unloading of the material was carried out from the KAMAZ-6522 dump truck with a payload capacity of 13 tons. Granulometric composition (in minus) was as follows: $0.4 \mathrm{~m}-100 \%, 0.3 \mathrm{~m}-50 \%, 0.1 \mathrm{~m}-20 \%$.

At the initial time from the area above the body program, the material is loaded by generating particles with a certain loading intensity and granulometric composition. After loading and some pause, lifting of the truck body takes place - the material is unloaded into the intermediate hopper and then to the hopper car, while in the calculated area a green parallelepiped is used to calculate the impact of the moving bulk material on the initially fixed air with the corresponding physical characteristics (density, viscosity and etc.). In this case, 


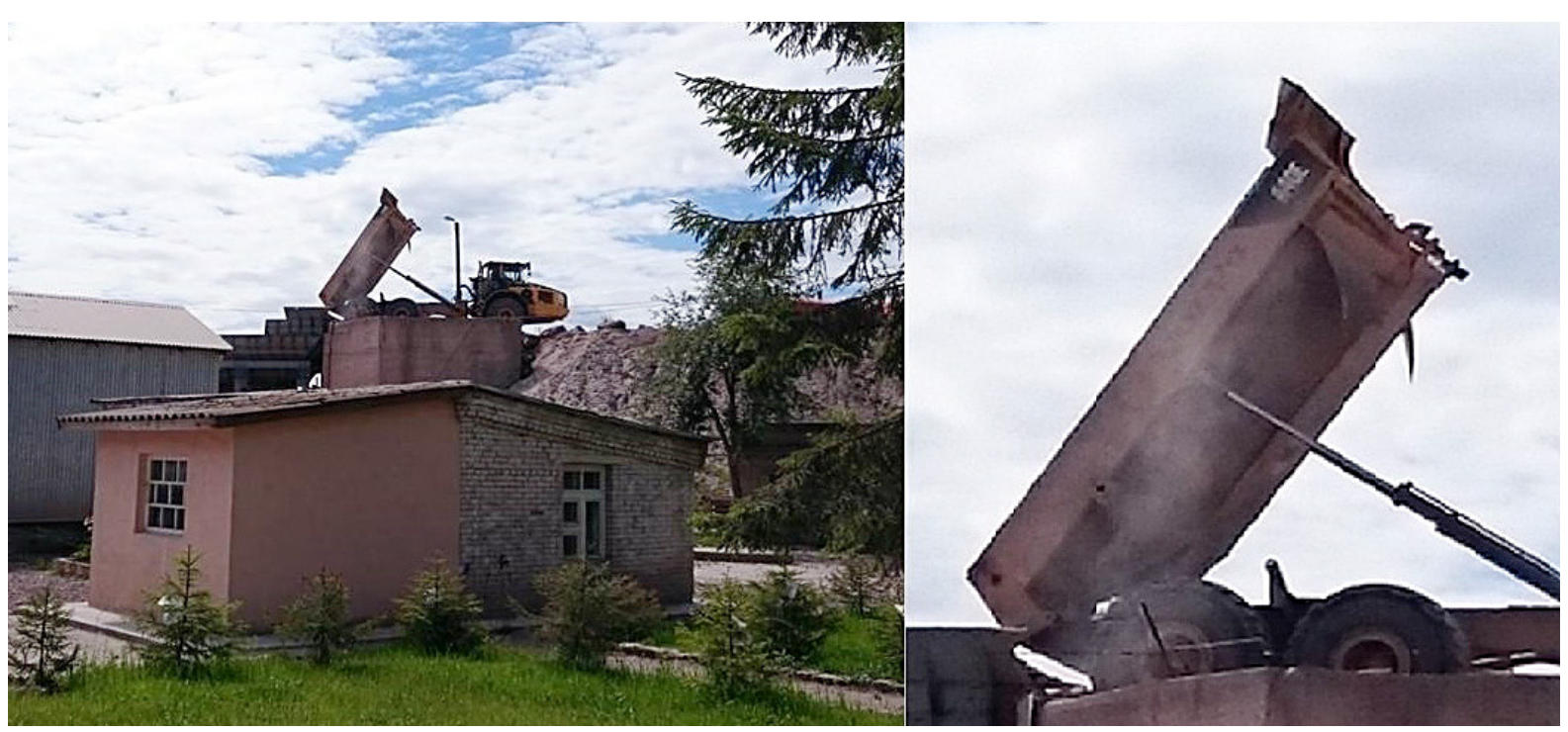

Figure 1. Dusting during the process of pouring of minerals at the factory "Vyborg Career Management"

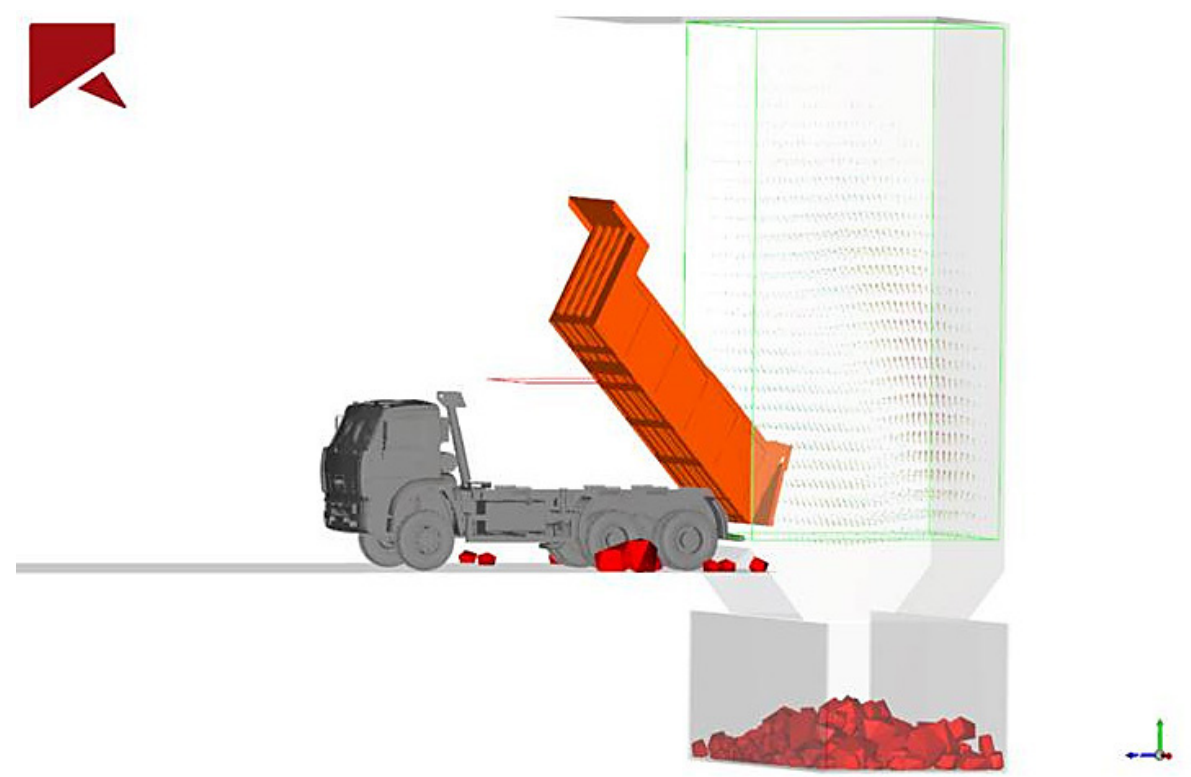

Figure 2. A simulated situation when unloading bulk material from the KAMAZ-6522 dump truck

the flow of geometric objects around the air is not taken into account (only the effect of the particle-the air medium) is calculated. The air velocity vectors in the calculated area are displayed (Figure 3). In turn, the speed of air movement can be interpreted as the volume of dust emission and dust transfer.

Additionally, it is possible to build various graphs of the dependencies of the parameters at each of their points examined based on the simulation data. According to the presented figure, the dependence of the maximum velocity of the air movement in the entire calculated volume on time was determined (Figure 4).
The maximum values of such velocities were observed from 10 to 14 seconds. Starting from 16 seconds to 25 seconds, the air velocity decreases almost to zero, which in fact can indicate the cessation of dust emission from the surface of the material poured and the beginning of the dust deposition process. Interpretation of the velocities of air movement into the concentration of aerosol particles was carried out using the dependencies presented by Ilchenkova [2005].

In the unloading zone of the mineral at the "Vyborg Career Management" enterprise, a series of full-scale studies was conducted to determine the speed of air movement provoked by the pro- 


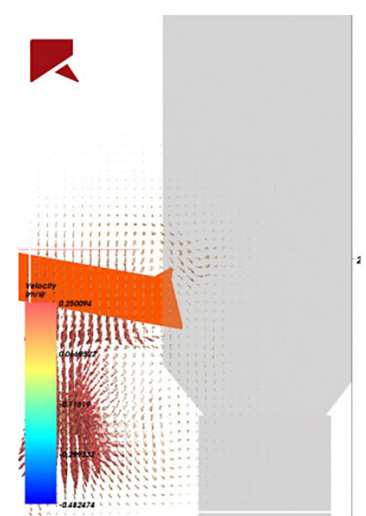

$7 \mathrm{sec}$

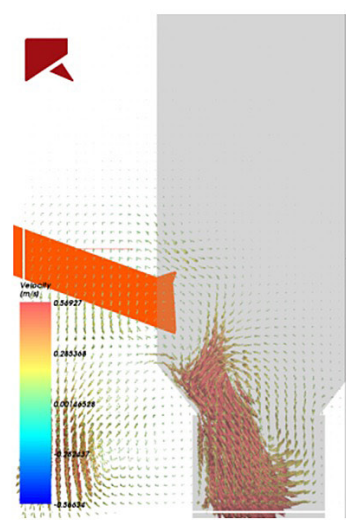

$9 \mathrm{sec}$

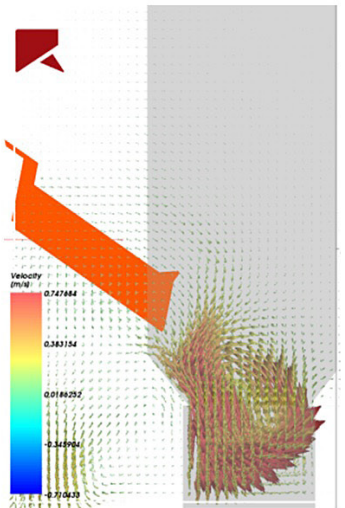

$12 \mathrm{sec}$

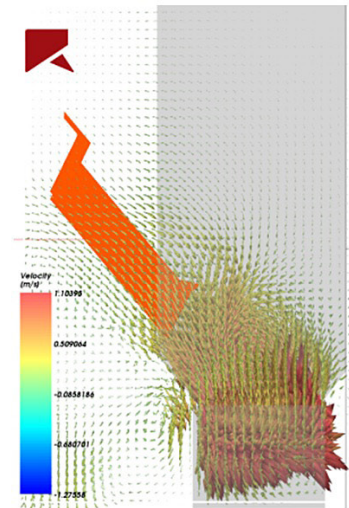

$15 \mathrm{sec}$

Figure 3. Distribution of air and particle velocities when material is poured

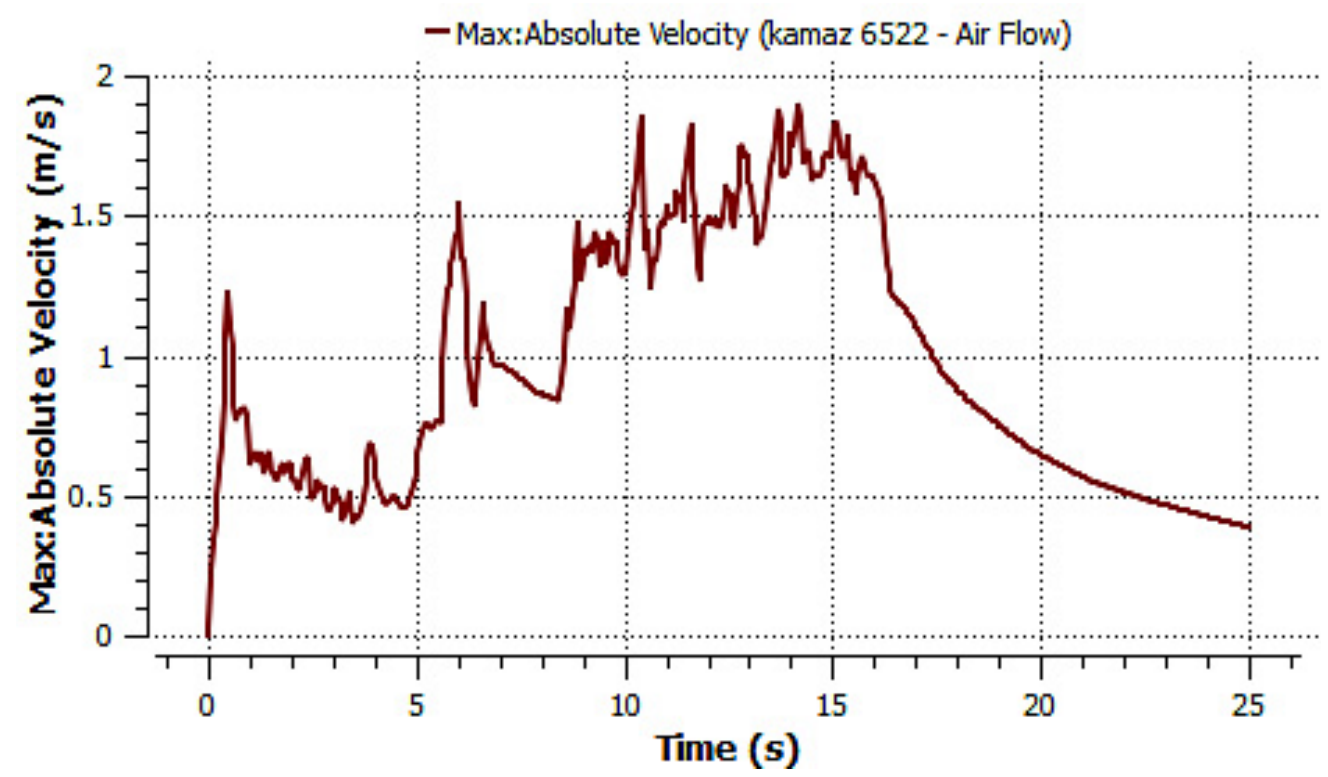

Figure 4. Dependence of the maximum velocity of air in the entire calculated volume on time

cess of pouring and concentration of dust in the air. When comparing the results, the convergence was of the order of $18 \%$, which can be considered acceptable for the conditions of complex models.

The model built with the use of "Ansys" and "Rocky" software packages allows to solve a number of urgent problems:

1. Determination of the parameters of insulating elements (casings, insulating bunkers, other protective structures) in order to optimize the movement of dust streams for their accumulation and safe disposal;

2. The choice regarding the location, speeds and heights of conveyor belt sections, the choice of operating modes of rail and road transport in order to minimize the release of solid suspended particles;
3. Perfection of the construction of devices for pouring and receiving bunkers;

4. Improvement of the effectiveness of dust suppression by optimizing the motion of dust streams;

5. Selection of the location and direction of dust suppression devices based on the data pertaining to the distribution of velocities and the number of suspended particles;

6. Reduction of the number of experimental samples of new developments by providing them with quality software models;

7. The operating mode of each individual dust suppression nozzle located at these points can be selected according to the graphs of the dependence of the air velocity on time at different points. 


\section{CONCLUSIONS}

The use of software complexes will allow modeling of the processes of separation and propagation of dust particles during the production processes at sites the impact assessment of which could previously be carried out using computational methods that do not take into account a number of external factors, and also through means of production monitoring. The software model, built with the use of "Ansys" and "Rocky" packages, allows solving a number of actual tasks in the production:

1. Determination of dominant directions and volume of dust emission at dusting objects;

2. Identification of unpredictable vectors of dust transfer in the places of pouring of dust material: receiving hoppers of crushing equipment, points of overfilling on conveyors, railway and vehicle loading devices;

3. Simulation of sifting of dispersed particles of drill cuttings in the production of drilling and blasting and prospecting works;

4. Calculation of the dust extraction parameters from any dynamic surfaces taking into account the conditions of protection;

5. Calculation of the dust during the work in quarries and other open areas.

Thus, the use of new modeling tools in the mining industry, in the conduct of scientific and research work in this direction will significantly speed up the process of developing and implementing new devices, significantly improve the quality of existing equipment, as well as shorten the period of production testing for debugging and troubleshooting of the implemented devices.

\section{Acknowledgements}

This work was supported by the Government of St. Petersburg, a grant from the President of the Russian Federation and the Center for Collective Use of the St. Petersburg Mining University.

\section{REFERENCES}

1. Basov K.A. 2016. ANSYS for constructors. DMK Press, Russia, pp. 248.

2. Bulbashev A.P., Gasparian N.A., Kovshov S.V., Nikulin A.N., Smirnov Yu.D., Shuvalov Yu.V. 2009. Rational organization of mining in quarries with difficult working conditions of miners. St. Petersburg. International Academy of Ecology, Human and Nature Safety, pp. 464.

3. Fedorova N.N., Valger S.A., Danilov M.N., Zaharova U.V. 2017. Basics of working in Ansys 17. DMK Press, Russia, pp. 210.

4. Ilchenkova S. 2005. Reduced dust load on the environment by binding particulate materials dusty surfaces in the territory of mining enterprises. PhD thesis, pp. 213.

5. Ivanov A.V., Smirnov Yu.D. 2016. The impact of anthropogenic alluvial arrays on areas settlements depending on the particle size distribution of stored tailings. Journal of Ecological Engineering, 17(2), 59-63.

6. Kaplun B.A., Morozov E.M., Shamraeva M.A. 2017. ANSYS is in the hands of an engineer. A Practical Guide. Lenand Publishing, Russia, pp. 272.

7. Pashkevich M.A., Smirnov Yu.D., Korelsky D.S., Ivanov A.V. 2014. Dust suppression bunker. Patent for invention of the Russian Federation No. 2536573, IPC B65G69/18, applicant and patent holder of the Federal State Educational Establishment of Higher Professional Education "National Mineral Resources University", No. 2013131917/11; application on 07.09.2013; published on 27.12.2014, the Bulletin No. 36.

8. Pashkevich M.A., Smirnov Yu.D., Dobrynin O.S., Bulbashev A.A., Ivanov A.V. 2012. Sprayer for dust suppression. Patent for invention of the Russian Federation No. 2446021, IPC B05B7/12, applicant and patent holder of the "Mining University", No. 01010133351/03; application on 13.08.2010; published on 27.03.2012, Bulletin No. 9.

9. Volkodaeva M.V. 2015. The use of geoinformation technologies for optimization of the location of air quality monitoring stations. Journal of Mining Institute, 215, 107-114. 